\title{
PRELIMINARY DESIGN AND TESTING OF A SERVO-HYDRAULIC ACTUATION SYSTEM FOR AN AUTONOMOUS ANKLE EXOSKELETON
}

\author{
Emmanuel Viennet*, Loïc Bouchardy \\ HES-SO University of Applied Sciences and Arts Western Switzerland, Boulevard de Pérolles 80, 1700 Fribourg \\ * Corresponding author: Tel.: +41 264296670 ; E-mail address: Emmanuel.Viennet@hefr.ch
}

\section{ABSTRACT}

The work presented in this paper aims at developing a hydraulic actuation system for an ankle exoskeleton that is able to deliver a peak power of $250 \mathrm{~W}$, with a maximum torque of $90 \mathrm{~N} . \mathrm{m}$ and maximum speed of $320 \mathrm{deg} / \mathrm{s}$. After justifying the choice of a servo hydraulic actuator (SHA) over an electro hydrostatic actuator (EHA) for the targeted application, some test results of a first functional prototype are presented. The closed-loop unloaded displacement frequency response of the prototype shows a bandwidth ranging from $5 \mathrm{~Hz}$ to $8 \mathrm{~Hz}$ for displacement amplitudes between $+/-5 \mathrm{~mm}$ and $+/$ $20 \mathrm{~mm}$, thus demonstrating adequate dynamic performance for normal walking speed. Then, a detailed design is proposed as a combination of commercially available components (in particular a miniature servo valve and a membrane accumulator) and a custom aluminium manifold that incorporates the hydraulic cylinder. The actuator design achieves a total weight of $1.0 \mathrm{~kg}$ worn at the ankle.

Keywords: Exoskeleton; Ankle-foot orthosis; Hydraulic actuator; Wearable robotics; Rehabilitation

\section{INTRODUCTION}

Lower limb powered exoskeletons are an emerging technology that shows potential in rehabilitation therapy, for instance by improving functional recovery and psychological well-being after a stroke [1]. In a recent clinical study [2], powered ankle-foot exoskeletons have shown biomedical benefits compared to classical passive ankle-foot orthosis. However, despite the positive results, all participants of the study indicated that mass and size were concerns with using the powered exoskeleton on a daily basis. With high power-to-weight and force-to-weight ratios, hydraulic actuators are an interesting alternative to electric motors today in use in the vast majority of exoskeleton projects.

\section{GAIT CYCLE}

The gait cycle, shown in Figure 1, is divided into the stance and swing phases. During the stance

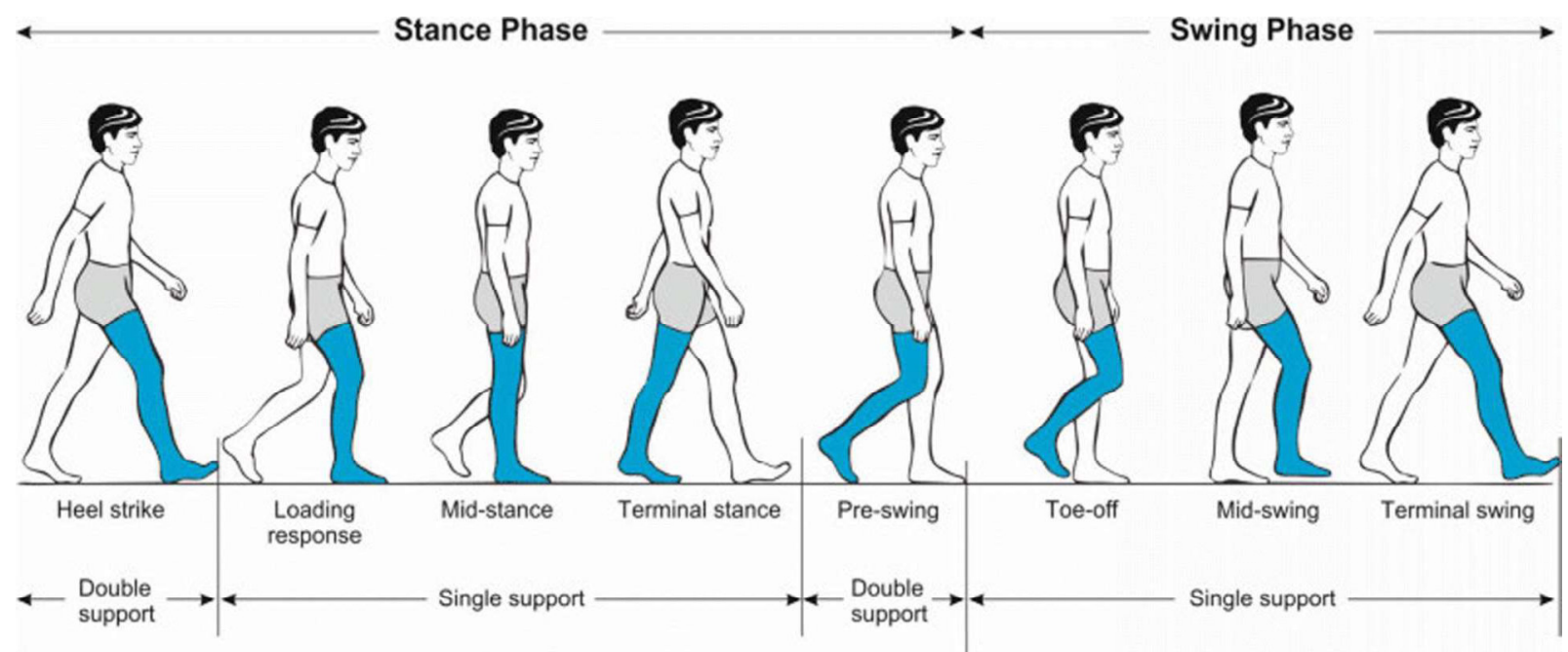

Figure 1: Phases of the normal gait cycle, reproduced from [3] 


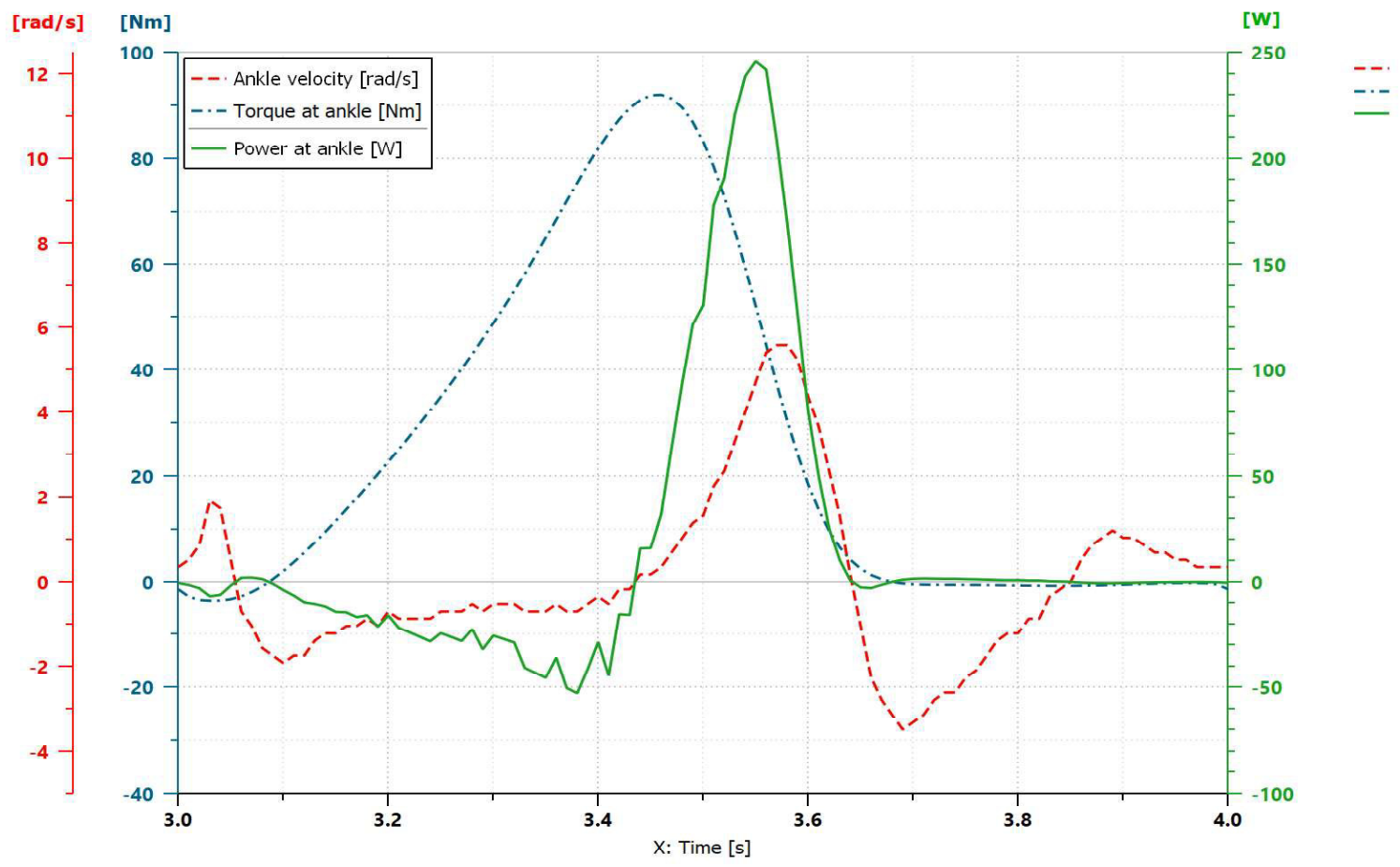

Figure 2: Angle velocity, torque and power at the ankle for $68 \mathrm{~kg}$ individual, reproduced from [4]

phase, the foot is in contact with the floor and the ankle is loaded. It actually receives power from the body during in the first half of the stance phase and provides power during the second half. During the following swing phase the ankle moves unloaded.

The working cycle of the ankle-foot joint depends on the walking conditions and the subject weight. The design presented in this paper is based on flat-floor normal-speed walking for a $68 \mathrm{~kg}$ individual as provided in [4] and reproduced in Figure 2.

The analysis of this working cycle provides key information for the design of the actuator:

- The power demand at the ankle is highly variable over the gait cycle. The positive part of this power has the form of a peak that lasts less than $20 \%$ of the total cycle and reaches a maximum value of $250 \mathrm{~W}$ for a $68 \mathrm{~kg}$ individual.

- There is a potential for recovering energy during the negative power phase. Besides, it occurs naturally for healthy subjects because mechanical energy is stored in the muscletendon system during this phase.

- A maximum velocity of $320 \mathrm{deg} / \mathrm{s}$ is reached during plantarflexion (foot down) and of 200 $\mathrm{deg} / \mathrm{s}$ during dorsiflexion (foot up)
- A maximum plantarflexion torque of 90 N.m is necessary (for $100 \%$ assistance) during the terminal stance phase (push-off) and a very limited dorsiflexion torque of less than 5 N.m is sufficient during heel strike.

With the help of the FFT shown in Figure 3, it appears clearly that, in addition to the maximum speed request, it is necessary to specify that the actuation system shall be able to track frequencies at least up to 4 or $5 \mathrm{~Hz}$ without major attenuation.

\section{ENVISAGED HYDRAULIC CONCEPTS}

While pioneering projects of hydraulic actuated exoskeletons like [5] used servo hydraulic actuation (SHA), two recent researches dealing with ankle-foot orthosis [6] and prosthesis [7] focussed on electro hydrostatic actuation (EHA). In this paper, we compare these two concepts by combining information provided on the datasheets of relevant components and results of numerical simulations.

For virtually assessing its performance, we propose a preliminary design of an EHA concept consisting of:

- E-motor EC 90 flat, 360 Watt, 24V, from Maxon Motors, Switzerland 
- Motor controller ESCON Mod. 50/5, from Maxon Motors, Switzerland

- Reversible axial piston pump with fixed displacement TFH-040 (414 $\left.\mathrm{mm}^{3}\right)$ from Takako, Japan

- Hydraulic hoses $1.5 \mathrm{~m}$ long, internal diameter $4 \mathrm{~mm}$ between pump and cylinder
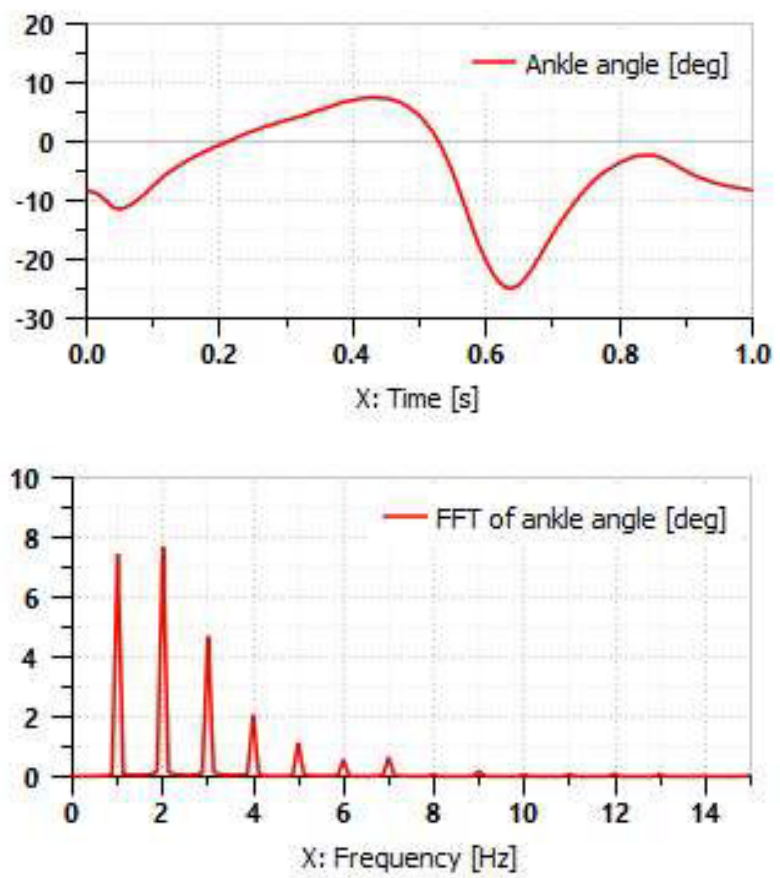

Figure 3: FFT of ankle angle during a gait cycle

We propose a corresponding preliminary design for a SHA concept as follows:

- E-motor EC frameless 60 flat, 100 Watt, 24V, from Maxon Motors, Switzerland

- Motor controller ESCON Mod. 50/5, from Maxon Motors, Switzerland

- Axial piston pump with fixed displacement PB-33.6 $\left(76 \mathrm{~mm}^{3}\right)$ from Hydroleduc, France

- Hydraulic hoses $1.5 \mathrm{~m}$ long, internal diameter $4 \mathrm{~mm}$ between pump and valve block

- Membrane accumulator AC13-1/4 (13 $\mathrm{cm}^{3}$, 250 bar operating pressure) from HAWE, Germany

- Servovalve E024 Super low leakage from Moog, England

- No hose between valve block and cylinder

The hydraulic schematics of the two concepts are given in Figure 4 and Figure 5. In both cases, a $10 / 8$ hydraulic cylinder with $40 \mathrm{~mm}$ stroke and 250 bar operating pressure is considered even if such a cylinder is not found on the market.
We modelled and simulated the two concepts in order to compare their ability in tracking a prescribed sinusoidal displacement of the cylinder rod with an amplitude of $+/-5 \mathrm{~mm}$ at different frequencies. The results are presented in form of a Bode diagram in Figure 6.

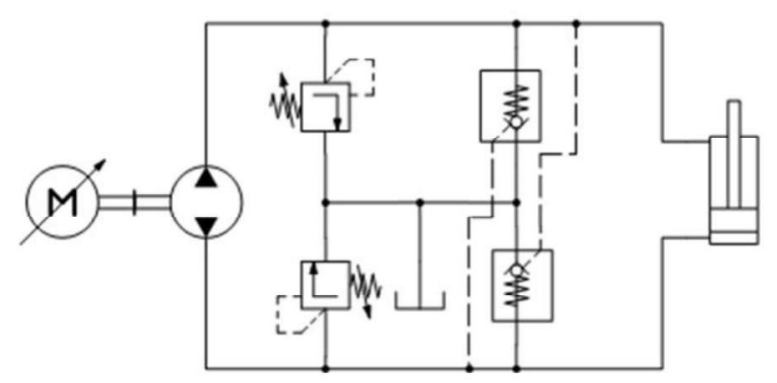

Figure 4: EHA

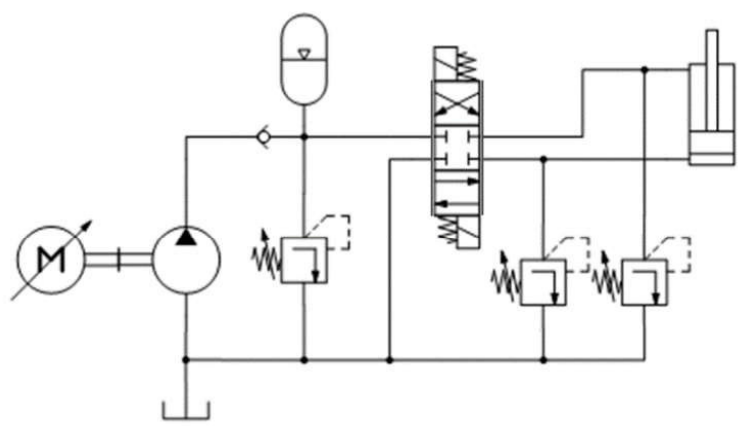

Figure 5: SHA

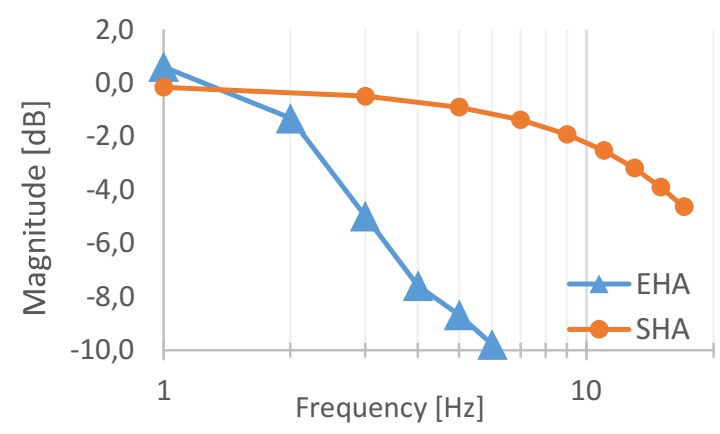

Figure 6: Simulated closed-loop unloaded frequency response for $+/-5 \mathrm{~mm}$ position

The $-3 \mathrm{~dB}$ cut-off frequencies are simulated respectively below $3 \mathrm{~Hz}$ for the EHA system and above $12 \mathrm{~Hz}$ with the SHA system. This makes a big advantage for the SHA system in terms of dynamics, especially when recalling that the typical angle profile during walking involves significant frequency contents up to $5 \mathrm{~Hz}$. 
One important reason for EHA to show lower dynamic performance than SHA is due to the time needed to build up the pressure in the hydraulic hoses. In the performed simulations, the oil bulk modulus was that of a mineral oil with $1 \%$ entrained air.

Another criterion for comparison is the weight worn at the ankle that we evaluated and summarized in Figure 7 (weight of the actuator only, excluding the structure of the exoskeleton). The values with the mention "estimated" are based on the preliminary design presented previously and shall be considered as ambitious yet realistic targets. The value with the mention "actual" is the result of a first-iteration detailed design presented in section 6 of this paper.

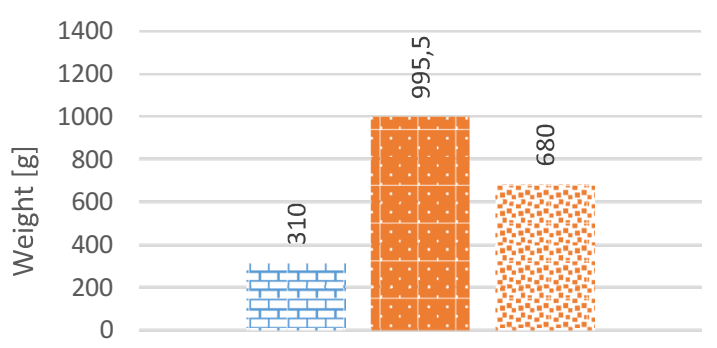

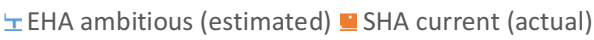

SHA ambitious (estimated)

Figure 7: Comparison of weight worn at the ankle for EHA and SHA, mix of actual and estimated values

Finally, choosing between EHA and SHA is a choice between two conflicting objectives namely dynamics versus light weight - that is inevitably frustrating in applications like exoskeletons where both are equally important.

\section{FIRST FUNCTIONAL PROTOTYPE}

In the presented project, we decided to develop a SHA concept, thus ensuring sufficient dynamic performance at the price of more effort to be put into weight reduction.

We built a first functional prototype with the goal of better specifying the further development. This functional prototype is based on the Moog servo valve E024 and the Hawe accumulator AC13 already mentioned and illustrated in the Figure 8 next to a coin of five Swiss francs. A tailored steel manifold was machined to accommodate a miniature check valve
CHFA1566501A from The Lee Company, USA and a high pressure filter QG0127 from Pall Corporation, USA. The manifold also interfaces pressure sensors 3100 from Gems, USA, and standard pressure relief valves.

Due to the lack of hydraulic cylinders commercially available in the needed power range, we used a LB6 $080500504 \mathrm{M}$ differential cylinder from Hawe-Hoerbiger, Germany, that was smaller than initially designed (diameters 8 $\mathrm{mm} / 5 \mathrm{~mm}$ ) and limited to 160 bar. For practical reasons, short hoses of diameter $4 \mathrm{~mm}$ were used to connect the cylinder to the manifold.

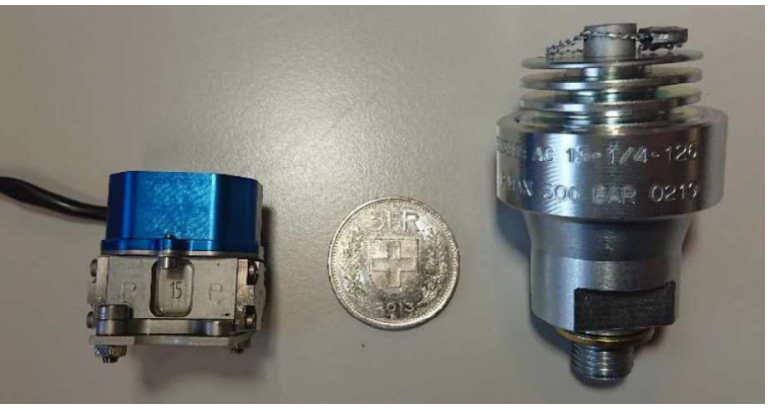

Figure 8: Moog E024 servo valve and Hawe 13cc accumulator used for functional prototype

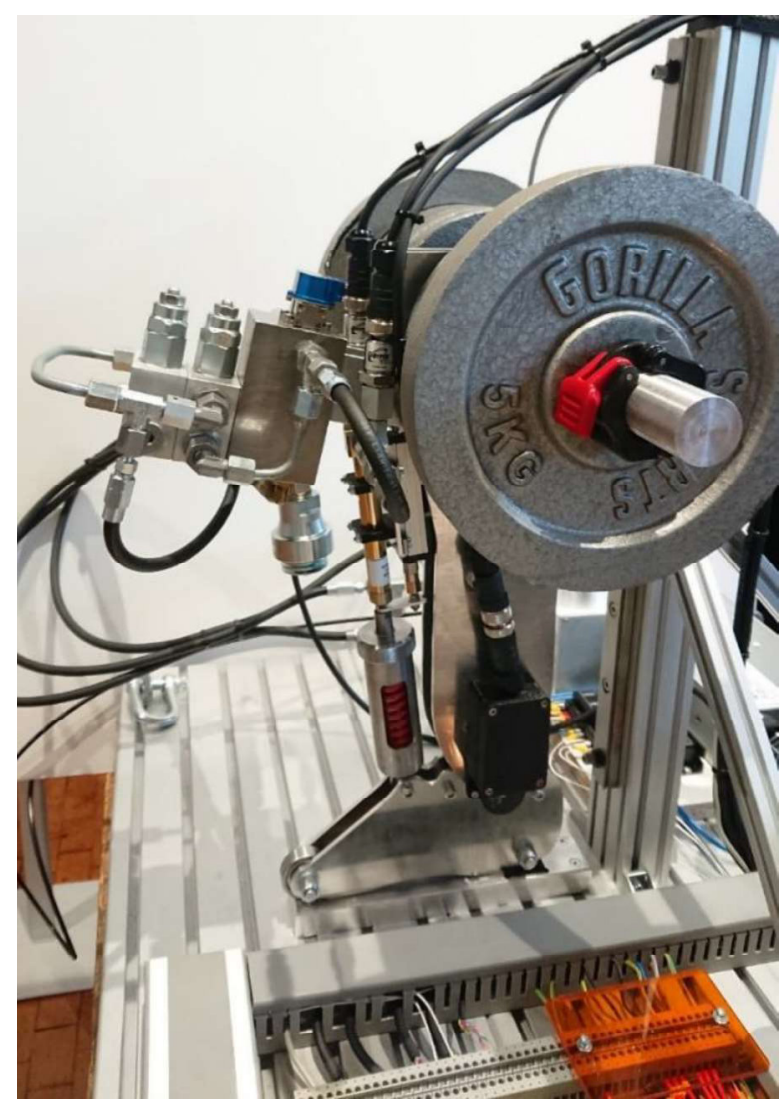

Figure 9: Functional prototype mounted on a test bench aiming at reproducing ankle motion under load (max. $35 \mathrm{~kg}$ load) 
The functional prototype was supplied with a constant pressure of 160 bar generated by a hydraulic power unit of $750 \mathrm{~W}$. It was mounted on a test bench illustrated in the Figure 9 whose goal is to allow the realisation of the ankle motion under load. The hydraulic cylinder is placed in front of the leg in order to use the higher force available during cylinder extension for the plantar flexion. The cylinder force is converted into a torque through a $50 \mathrm{~mm}$ long lever arm. It should be noted that both cylinder extension and retraction occur under load on the test bench, whereas during a real gait cycle, only cylinder extension would occur under load. For investigating the advantage of a series elastic actuation (not presented here) an encapsulated spring can be mounted in series between the cylinder rod and the "foot" of the test bench (visible in Figure 9).

\section{PRELIMINARY TEST RESULTS}

A position control loop is implemented with the help of a portable controller ChipKit uC32 from National Instruments / Digilent, USA. The control loop can be realised either on the hydraulic cylinder displacement or on the foot angle. We used a PI controller with different gains depending on the sign of the error, accounting for the inherent non-symmetrical behaviour of the differential cylinder and we implemented a Butterworth low-pass filter of $2^{\text {nd }}$ order with a cut-off frequency of $50 \mathrm{~Hz}$ on the sensor inputs (linear displacement and angle). During the performed tests, the frequency rate of the controller settled at approx. $1 \mathrm{kHz}$.

The magnitude of the closed-loop unloaded displacement frequency response of the prototype servo hydraulic actuator is shown in Figure 10. The measured $-3 \mathrm{~dB}$ cut-off frequency ranges from about $5 \mathrm{~Hz}$ for large displacement amplitudes $(+/-20 \mathrm{~mm})$ up to $8 \mathrm{~Hz}$ for small displacements $(+/-2.5 \mathrm{~mm})$. The measured cutoff frequency is lower than the simulated one (see Figure 6 for comparison). It is attributed to friction effects in cylinder at high speed and to compressibility effects, especially in the additional hoses used between the servo valve and the hydraulic cylinder.

The closed-loop position step responses given in Figure 11 were obtained with the gains of the controller being tuned for an overshoot below $10 \%$. It resulted in a maximum angular speed of about $400 \mathrm{deg} / \mathrm{s}$. With controller gains tuned for critical damping (results not presented here), a settling time of about $0.15 \mathrm{~s}$ was achieved for a $40 \mathrm{deg}$ step, i.e. a three times faster response than the one obtained with an EHA in [6].

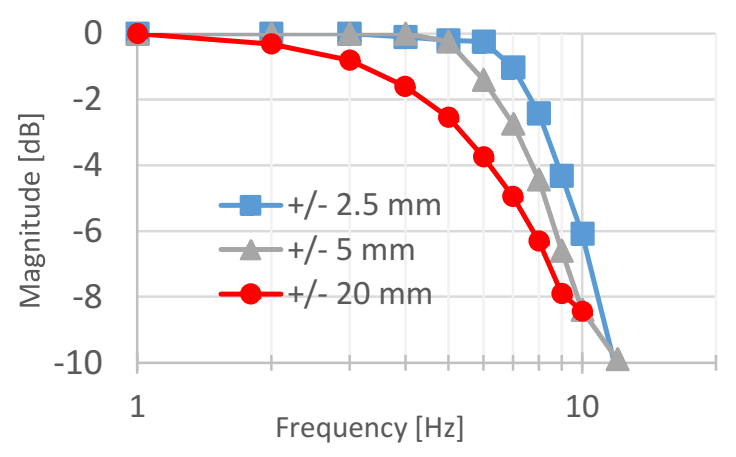

Figure 10: Measured SHA closed-loop unloaded displacement frequency response
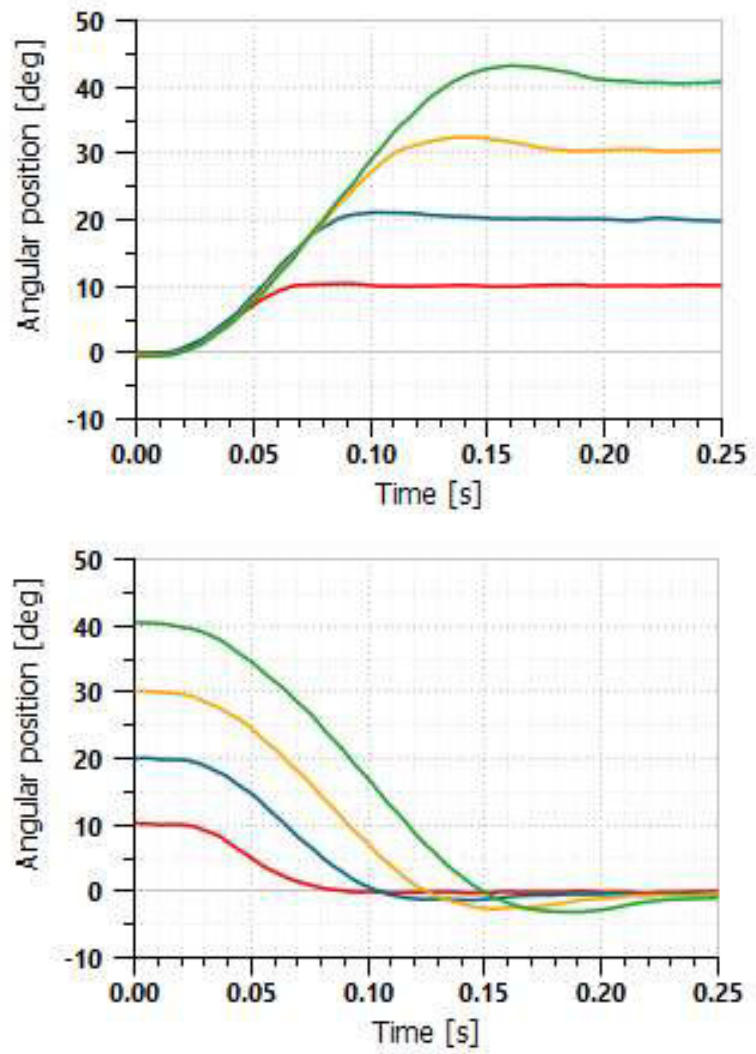

Figure 11: Measured SHA closed-loop PI control unloaded position step responses

\section{NEXT STEPS}

The test results obtained with the first functional prototype confirmed the adequate dynamic characteristics of the SHA for the application. As an answer to the lack of available hydraulic cylinders suited for our application and to the 
need for weight optimization, a tailored aluminium hydraulic manifold was designed to integrate all necessary components of the SHA, including the hydraulic cylinder. An exploded view of the design is given in Figure 12.

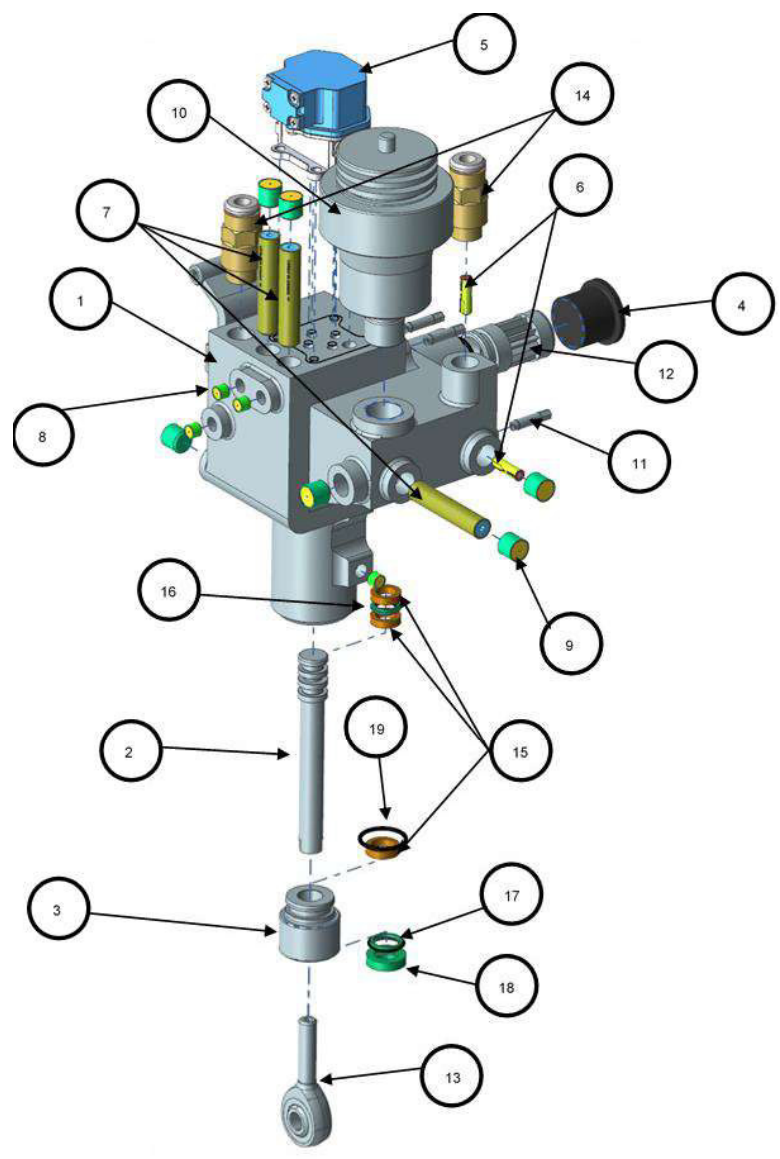

Figure 12: Exploded view of the integrated SHA under development

The overall weight of the actuator is just below $1.0 \mathrm{~kg}$ and the detailed weights of the components are listed in Table 1. The heaviest parts are the hydraulic manifold and the accumulator weighting respectively $480 \mathrm{~g}$ and $300 \mathrm{~g}$. In the course of this project, only conventional machining techniques were considered for the manifold and not every materials were regarded. However, with the use of $3 \mathrm{D}$ metallic printing techniques and advanced materials, we assess that the weight of the hydraulic manifold can be still reduced by $35 \%$. In addition to that, by a better integration of the accumulator in the hydraulic manifold (only possible with the support of the supplier or by developing in-house a specific accumulator) a further reduction of approx. $150 \mathrm{~g}$ would be possible. Combining all these efforts, an optimized weight of $680 \mathrm{~g}$ can be achieved, as indicated in Figure 7.

Table 1: Weight of SHA components

\begin{tabular}{llll}
\hline$\#$ & Component & Amount & Mass [g] \\
\hline 1 & Hydraulic manifold & 1 & 477 \\
2 & Cylinder rod & 1 & 20.3 \\
3 & Cylinder cap & 1 & 9.9 \\
4 & Plug for filter & 1 & 7.7 \\
5 & Servo valve & 1 & 95 \\
6 & Checkvalve & 2 & 0.7 \\
7 & Pressure limiter & 3 & 6.0 \\
8 & Plug 0.187 & 5 & 0.51 \\
9 & Plug 0.312 & 6 & 2.5 \\
10 & Accumulator & 1 & 300 \\
11 & Pressure sensor & 3 & 3.1 \\
12 & Filter & 1 & 9.0 \\
13 & Ball-head & 1 & 18 \\
14 & Connectors & 2 & 5.0 \\
15 & Guide band & 3 & 0.2 \\
16 & Piston seal & 1 & 0.2 \\
17 & Rod seal & 1 & 0.3 \\
18 & Wiper ring & 1 & 1.0 \\
19 & O-ring & 1 & 0.1 \\
Total mass & & $\mathbf{9 9 5 . 5}$ \\
\hline
\end{tabular}

\section{CONCLUSION AND OUTLOOK}

The main contribution of this paper is that we have designed a hydraulic actuator for an autonomous ankle exoskeleton that is able to provide full assistance during the gait cycle of a $68 \mathrm{~kg}$ individual at normal walking speed (max torque of 90 N.m and max. speed of $320 \mathrm{deg} / \mathrm{s}$ at the ankle). By combining results of both numerical simulations and preliminary tests, we have oriented ourselves toward a servo hydraulic actuation (SHA) rather than an electro hydrostatic actuation (EHA) because of its higher dynamic performance. A first design iteration has led to a weight just below $1.0 \mathrm{~kg}$ for the whole actuator and a potential for reducing this weight further down to $680 \mathrm{~g}$ has been identified. With a total target weight of less than $1.0 \mathrm{~kg}$ worn at the ankle, this would give us $320 \mathrm{~g}$ for the exoskeleton structure for which a very first concept is illustrated in Figure 13. 


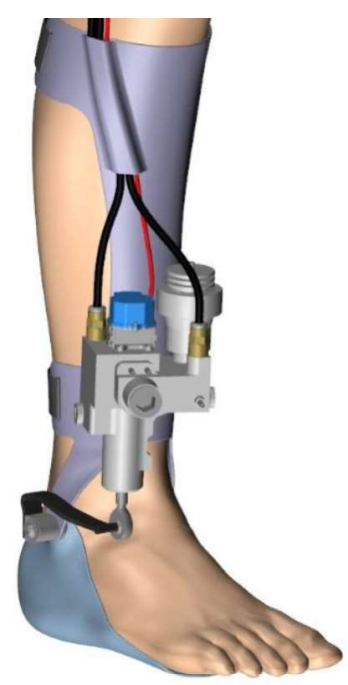

Figure 13: First concept for the integration of the actuator on an exoskeleton structure

\section{REFERENCES}

[1] Calabrò RS, Reitano S, Leo A, De Luca R, Melegari C, Bramanti P (2014) Can robotassisted movement training (Lokomat) improve functional recovery and psychological wellbeing in chronic stroke? Promising findings from a case study. Functional neurology, 29(2), 139_ 141.

[2] Russell Esposito E, Schmidtbauer KA, Wilken JM (2018) Experimental comparisons of passive and powered ankle-foot orthoses in individuals with limb reconstruction. J Neuroeng Rehabil. 2018 Nov 21;15(1):111

[3] Pirker W, Katzenschlager R (2017) Gait disorders in adults and the elderly: A clinical guide. Wien Klin Wochenschr. 2017 129(34):81-95

[4] Bovi G, Rabuffetti M, Mazzoleni P, Ferrarin M. (2010). A multiple-task gait analysis approach: Kinematic, kinetic and EMG reference data for healthy young and adult subjects. Gait Posture jan 2011 ; 33(1):6-13

[5] Zoss A, Kazerooni H, Chu A (2005) On the mechanical design of the Berkeley Lower Extremity Exoskeleton (BLEEX). IEEE/RSJ International Conference on Intelligent Robots and Systems, 2005, pp. 3465-3472

[6] Neubauer BC (2017) Principles of Small-Scale Hydraulic Systems for Human Assistive Machines. PhD thesis, University of Minnesota

[7] Yu T (2017) Actuation and Control of Lower Limb Prostheses. PhD thesis, University of Bath 